\title{
Automatic detection of lexical change: an auditory event-related potential study
}

\author{
Alexandra Muller-Gass, Anja Roye, Ursula Kirmse, Katja Saupe, Thomas Jacobsen and Erich Schröger \\ Institute for Psychology I, University of Leipzig, Leipzig, Germany \\ Correspondence to Dr Alexandra Muller-Gass, Institut für Psychologie I, Universität Leipzig, Seeburgstr. 14-20, 04I03, Leipzig, Germany \\ Tel: + 4934197359 83; fax: + 4934197359 69; e-mail: muller-gass@uni-leipzig.de
}

\begin{abstract}
We investigated the detection of rare task-irrelevant changes in the lexical status of speech stimuli. Participants performed a nonlinguistic task on word and pseudoword stimuli that occurred, in separate conditions, rarely or frequently. Task performance for pseudowords was deteriorated relative to words, suggesting unintentional lexical analysis. Furthermore, rare word and pseudoword changes had a similar effect on the event-related potentials,
\end{abstract}

starting as early as $165 \mathrm{~ms}$. This is the first demonstration of the automatic detection of change in lexical status that is not based on a co-occurring acoustic change. We propose that, following lexical analysis of the incoming stimuli, a mental representation of the lexical regularity is formed and used as a template against which lexical change can be detected.

Keywords: auditory event-related potentials, automaticity, lexical analysis, lexical categorization, lexical oddball paradigm, spoken word

\section{Introduction}

The detection of acoustic change is contingent on the formation of a sensory memory template that encodes the invariant information (i.e. the constancy) in the auditory environment. A violation of this invariance is then noted through a memory comparison process of the well-formed regular 'standard' representation and the incoming rare 'deviant' representation of the stimulation. This phenomenon is best studied using the oddball paradigm, in which a train of standard stimuli (that share some stimulus property) is rarely interspaced by a deviant stimulus (that deviates from the standards on this property). The detection of acoustic change is an automatic process, as it occurs irrespective of whether the change is relevant to a given task or not, and even when the stimulation occurs outside the focus of attention. This process is reflected by an eventrelated potential (ERP), the mismatch negativity (MMN) [1]. The preattentive detection of acoustic change may trigger an involuntary attention-switching mechanism and lead to performance deterioration on a concurrent primary task [2].

Although task-irrelevant change detection has been extensively studied using acoustic violations of regularity, it is not known whether the brain can also automatically detect a change in a higher-order stimulus property when it does not coincide with acoustic change. To address this question, we examined whether ERPs and behavioral data reveal the detection of a task-irrelevant change in the lexical status of a stimulus. Recently, several ERP studies have demonstrated that the lexicality of a stimulus modulates the acoustic change detection process, and this, even during a passive listening situation. Typically, in these studies, a tobe-ignored word and a slightly dissimilar pseudoword serve, in separate conditions, as standard and deviant stimuli; in this way, the acoustic contrast between the standard and the deviant is kept constant across conditions. Pulvermüller and colleagues [3-6] have repeatedly reported that the MMN is enhanced when the deviant formed a word compared to a pseudoword. They proposed that this lexical MMN enhancement is due to the activation of long-term memory (LTM) traces for words that occur in addition to the acoustic change detection-related activation. Findings from other studies $[7,8]$ have, however, suggested that lexical analysis is performed regardless of the occurrence of a concurrent acoustic change (i.e. also for the standard stimulus). Jacobsen et al. [7] found a lexical MMN enhancement when the standard was a word as compared to a pseudoword. They suggested that the sensory memory representation of the standard was more elaborate for words than for pseudowords, making the detection of deviance more salient. In contrast, Diesch et al. [8] showed that the MMN was enhanced when a pseudoword served as the standard, perhaps because additional prelexical processes, triggered by the failed lexical activation for the pseudoword, benefited the encoding of its memory representation. In a similar vein, other higher-order properties of nonspeech stimuli, such as their familiarity or semantic valence, have also modulated the acoustic detection of change (i.e. the $M M N)$, and even the extent of the subsequent involuntary attention-switch process. These effects have been attributed to the familiarity/meaningfulness of both the deviant [9-11] and the standard [10] stimulus.

Together, these results suggest that task-irrelevant stimuli undergo some form of LTM analysis nearly simultaneously with basic sensory analysis; they have, however, only demonstrated that the activation in LTM has a modulatory effect on the stimulus representation within sensory memory. In a recent functional MRI study that used a passive lexical oddball task, Shtyrov et al. [12] found 
change-related activation following a deviant word but not a deviant pseudoword, although the acoustic contrast between the word and pseudoword was identical in the two conditions. They concluded that the change-related activation could thus only be owing to lexico-semantic processing (and not to acoustic change detection), and hypothesized that the detection of acoustic and lexical change may actually be subserved by two distinct processes. In this study, we unconfound the effects of lexical change from those related to acoustic change. To achieve this, we ensured that any physical invariance that could be extracted from the standards would not be violated by the deviants. For this purpose, we employed a set of 34 disyllabic words and constructed 34 pseudowords by systematically recombining the initial syllable of each word with the terminal syllable of a different word. Participants could thus only distinguish words from pseudowords based on lexicality, and this, at the earliest after the onset of the terminal syllable. The lexical status of the stimuli was, however, taskirrelevant as participants performed a task based on a physical (nonlinguistic) aspect of these stimuli. In separate conditions, words and pseudowords served as standards and deviants. A comparison of these conditions may reveal whether physically identical stimuli are processed differently when they represent a rare lexical change. Our paradigm further affords the possibility to examine whether a task-irrelevant lexical change interferes with performance on the nonlinguistic task.

\section{Methods \\ Participants}

Fourteen adults (nine women) with a mean age of 23.6 years $(\mathrm{SD}=2.6$ years) volunteered for this study. All participants were right-handed, reported normal hearing and were native speakers of German. Written informed consent was obtained before the experiment and an honorarium was offered as compensation. The experiment was approved by an ethics committee of the Universität Leipzig. The data of one extra participant were rejected because they contained an excessive electroencephalographic artifact. Moreover, three extra participants were dismissed after the practice session owing to poor performance on the primary task.

\section{Stimuli}

The auditory stimuli were disyllabic words $(n=34)$ and pseudowords $(n=34)$ uttered by a female native speaker of German [Words and pseudowords were easily discriminable. When a subsample of participants $(n=7)$ were asked to overtly categorize these stimuli, a mean hit rate of 0.96 and reaction time of $530 \mathrm{~ms}$ (measured from onset of the second syllable) was achieved]. The words were chosen from the Celex database with the following stipulations: they had a high frequency of occurrence in the German language (lemma frequency); they consisted of two consonant-vowelconsonant syllables; and the primary stress was on the initial syllable. Each word had a corresponding pseudoword, such that they shared an identical initial syllable. The pseudowords were further constructed by combining the initial syllable with the terminal syllable of a different word (e.g. word-pseudoword pair: Zirkus-Zirtel; Wimper-Wimkus). As such, all 34 initial and 34 terminal syllables figured in both word and pseudoword categories.
Several exemplars of the words and pseudowords were recorded in a sound-proof room. The actual stimuli were then selected based on the duration of, and perceived similarity between, the initial syllable of a corresponding word-pseudoword pair [It was verified in a subsample of participants $(n=7)$ whether lexical status of the stimuli could be determined on the basis of the initial syllable. The mean hit rate on this task was close to chance level (mean $=0.57$, $\mathrm{SD}=0.11)]$. The stimuli were subsequently low-pass filtered with a cutoff frequency of $4.8 \mathrm{kHz}$, and normalized to the same loudness by equating peak sound energy. Mean stimulus duration was $769 \mathrm{~ms}$ for words, and $772 \mathrm{~ms}$ for pseudowords, with the initial syllable lasting 319 and $335 \mathrm{~ms}$, respectively. For the purpose of the task, the stimuli were subsequently manipulated so that in one instance, they ended in an upward frequency glide, whereas in another, in a downward glide. The glide started with the onset of the vowel of the terminal syllable, and gradually increased from a 0 to a $10 \%$ stretch. All stimuli were delivered at $75 \mathrm{~dB}$ sound pressure level.

\section{Procedure}

To avoid learning effects during the experiment, participants were shown a list of all stimuli and asked to passively listen to them. During the practice session, participants classified the stimuli based on the direction of the frequency glide until their hit rate $\geq 0.75$.

During the experimental session, participants were seated in a sound-attenuated room. The stimuli were presented binaurally via headphones with a stimulus onset asynchrony that varied between 1850 and 2150 ms. Participants engaged in a forced-choice categorization task, indicating by button-press whether the stimulus ended in an upward $(P=0.5)$ or downward $(P=0.5)$ glide. This task was performed in two lexical context conditions: one in which the frequent standards $(P=0.85)$ were words and the infrequent deviants $(P=0.15)$ were pseudowords, and another with the reverse word-pseudoword probability of occurrence. The order of stimulus presentation was pseudorandomized with the constraint that each deviant was separated by at least two standards.

Every block included the 34 items from the standard category and six randomly selected items from the deviant category; each of these items was presented thrice, for a total of 120 trials per block. Eight blocks were presented for each of the two lexical context conditions (in an alternating manner), for a total of 960 stimuli per condition. The response-to-key mapping and the order of conditions were counterbalanced across participants.

\section{Electroencephalogram/event-related potential recording} Electroencephalographic activity was continuously recorded with $\mathrm{Ag}-\mathrm{AgCl}$ electrodes from five scalp sites $(\mathrm{Fz}, \mathrm{Cz}, \mathrm{Pz}, \mathrm{FC} 3, \mathrm{FC} 4)$ and from left and right mastoids. The reference electrode was placed on the tip of the nose. Horizontal and vertical electrooculographic activity was recorded from electrodes placed lateral to the outer canthus of each eye, and at the infraorbital and supraorbital ridges of the right eye, respectively. Interelectrode impedances were below $5 \mathrm{k} \Omega$.

The physiological signals were digitized at a $500-\mathrm{Hz}$ sampling rate (filter bandpass: $0.1-100 \mathrm{~Hz}$ ) and subsequently filtered offline with a bandpass of $1-15 \mathrm{~Hz}$. The 
continuous data were reconstructed into discrete $1100 \mathrm{~ms}$ epochs, including a -100 to $0 \mathrm{~ms}$ baseline time-locked to the onset of the terminal syllable. Epochs containing signal changes exceeding $100 \mu \mathrm{V}$ on any channel were excluded from further analyses. The single-participant ERPs were averaged for stimulus type (standard, deviant) and lexical status (word, pseudoword). Only correctly classified trials were included in the ERP averages.

\section{Data analysis}

Performance on the glide classification task was determined separately for words and pseudowords when they served as standards and deviants. A response occurring 300-1950 ms from the onset of the terminal syllable was classified as a 'hit'. The mean reaction time was computed by averaging only those trials that included correctly classified stimuli.

In both conditions, the deviant ERP included an additional positive-negative complex relative to the standard ERP. As a result of interparticipant latency variation, maximum peak amplitude detection methods were used to score the amplitude of the positivity in the $100-200 \mathrm{~ms}$ time window. The amplitude of the negative deflection was quantified as the average of all data points within $\pm 20 \mathrm{~ms}$ of the maximum peak latency in the grand-average waveform.

Performance measures were subjected to two-way analyses of variance with repeated measures on stimulus type and lexical status. ERP data were analyzed via three-way repeated measures (stimulus type, lexical status, electrode site) analyses of variance.

\section{Results}

\section{Behavioral data}

Stimulus type (standard, deviant) did not affect performance measures; both the main effect and the interaction involving this factor were not significant. The main effect of lexical status (word, pseudoword) was significant for reaction time and hit rate, $\mathrm{F}(1,13)=18.78, P<0.001$ and $\mathrm{F}(1,13)=13.03, P<0.01$, respectively. Participants were faster and more accurate in classifying the frequency glide in words than in pseudowords (reaction time: 690.2 vs. $714.6 \mathrm{~ms}$; hit rate: 0.83 vs. 0.80 ).

\section{Event-related potential data}

As seen in Fig. 1, the ERPs elicited by words and pseudowords showed a similar effect of stimulus type: the deviant ERPs were characterized by a positive and subsequent negative enhancement when compared with the standard ERPs. A significant main effect of stimulus type was found for both the peak amplitude of the positivity and the mean amplitude of the negative difference, $\mathrm{F}(1,13)=7.90, P<0.02$ and $\mathrm{F}(1,13)=5.55, P<0.04$, respectively. The positive enhancement was largest at about $165 \mathrm{~ms}$ (maximal at $\mathrm{Cz}$; mean $=0.6 \mu \mathrm{V})$, whereas the negative enhancement was largest at about $375 \mathrm{~ms}$ (maximal at $\mathrm{Pz}$; mean $=-0.7 \mu \mathrm{V}$ ). Neither the main effect of lexical status, of electrode site, nor the interactions involving these factors reached significance.

\section{Discussion}

This study investigated the automatic detection of rare taskirrelevant changes in lexical status. Our ERP data revealed that such changes were indeed detected, and this, whether rare words were presented among frequent pseudowords or

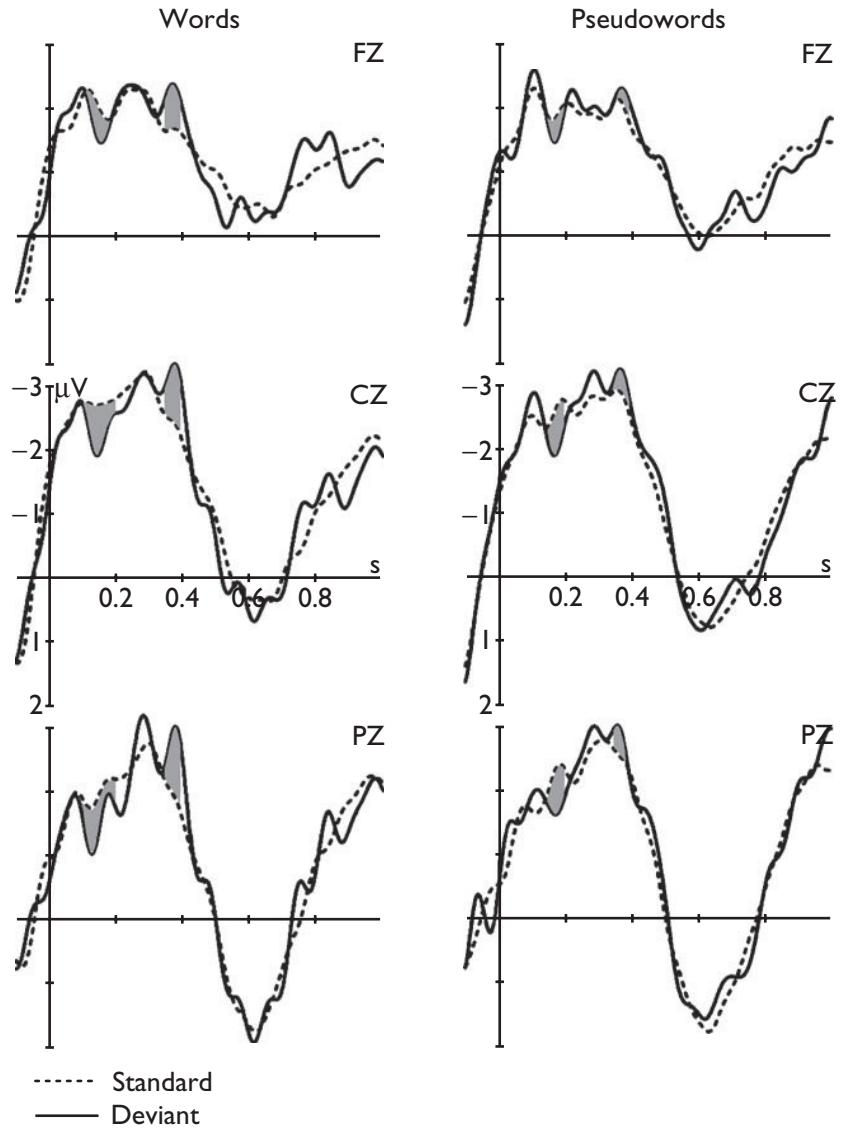

Fig. I Grand average ERPs elicited by words and pseudowords when they served as standards (dashed line) and deviants (solid line). The ERPs are averaged to the onset of the terminal syllable, the earliest point at which words and pseudowords could be differentiated. ERP, event-related potential.

rare pseudowords were presented among frequent words. Importantly, both the word and pseudoword deviants showed similar effects on the ERPs, which included a positive and a subsequent negative enhancement relative to the respective standard ERP. This deviance-related ERP effect could not be due to acoustic change detection as the lexical change did not signal a violation in the acoustical regularity of the stimulation (i.e. the acoustic variation among the standards was comparable with that between standard and deviant stimuli). Furthermore, this deviance effect could not be explained by a stronger activation of LTM memory traces for deviant words, as it was also visible following the deviant pseudowords. This finding is therefore inconsistent with the Shtyrov et al. [12] hypothesis that lexico-semantic processing of rare words, but not rare pseudowords, could occur independently of acoustic change detection; it does not, however, preclude the possibility that acoustic change detection may be modulated by the activation of the more extensive memory network underlying word stimuli [4].

The most parsimonious interpretation of the present data is that each stimulus underwent lexical analysis, and that a mental representation of regularity was established based on the lexicality of the standards, against which lexical change could be detected. Our behavioral data offer evidence that lexical analysis was indeed carried out even 
though it was not required to perform the nonlinguistic task: the frequency glide in standard and deviant words was categorized faster and more accurately than that in pseudowords. A similar performance effect for orally presented words and pseudowords has been shown during various linguistic tasks, such as lexical decision [13-15], phonetic categorization [15-17] and word repetition [18] tasks. Auditory lexical access is assumed to involve a matching process between the sensory input and a lexical representation. In contrast to words, pseudowords do not have a stored representation in the mental lexicon. As such, the delayed response to pseudowords during linguistic tasks is generally explained by a longer search for its (absent) lexical representation, which may weigh on the prelexical stages of speech processing. Implicit processing of lexicality has been demonstrated for visually presented words and pseudowords. In a positron emission tomography study, Price et al. [19] showed that both words and pseudowords activated the language network during a nonlinguistic visual feature detection task, with the activation being generally greater during presentation of pseudowords; behavioral performance on the detection task was, however, not affected by the lexical status of the stimulus.

The present results further suggest that lexical analysis culminated in the formation of a mental template that encoded the lexical status of the frequently occurring standards (i.e. the lexical context). In this way, a violation of the representation of lexical regularity would result in a similar brain response regardless of the lexical status of the standard and the deviant. Our ERP data, in fact, showed such a common response to lexical change. Deviant ERPs significantly diverged from standard ERPs at about $165 \mathrm{~ms}$, at which time a broadly distributed positivity was visible across the scalp. In studies employing lexical decision tasks with equiprobable words and pseudowords, ERP differences between these stimuli were observed 100-200 ms after the information allowing for lexical identification to become available [20,21]. It is therefore feasible that the early positivity in the present data represents the initial detection of lexical change. No such deflection is, however, visible to deviant stimuli that simultaneously represent a lexical and an acoustic change (e.g. [6-8]). This may be because the MMN to acoustic change occurs in a similar time window and thus overlapped this positivity; or alternatively, a representation of lexical regularity may not be established when only one item of a word or pseudoword category is employed as a standard stimulus, as was the case in these aforementioned studies. It is also possible that the elicitation of the lexical-change positivity requires participants to attend to the auditory stimulation. In the earlier studies, the acoustic/lexical change occurred during passive listening.

A later broadly distributed negative enhancement was also noted in our deviant ERPs, at about $365 \mathrm{~ms}$. Attias and Pratt [22] found a similar deviant ERP effect during an auditory lexical oddball task, which required participants to actively discriminate rare words (pseudowords) presented among pseudowords (words). Regardless of lexicality, both deviants (targets) and standards elicited a prolonged negativity from 100 to $400 \mathrm{~ms}$, a wave the authors called the 'sustained N400 (SN4)'. They suggested that the SN4 may reflect a specific lexical process engaged when a lexical or semantic decision is required; however, we found a similar sustained negative waveform to standards and deviants (in both lexical conditions), which indicates that a task requiring lexical processing is not necessary for its elicitation. Moreover, Attias and Pratt [22] related the enhancement of the SN4 (at about $400 \mathrm{~ms}$ ) for both deviant words and deviant pseudowords to the relationship between expectancy and N400, suggesting that it reflected processes associated with updating of a cognitive memory after the lexical identification of the unexpected deviant stimulus.

Our findings thus propose that a task-irrelevant change in lexical status is detected and may trigger further cognitive processing. The processing of the task-irrelevant change did not, however, interfere with task-relevant processing of a physical property of the stimulus: no performance deterioration on the glide categorization task was noted following the deviant relative to the standard stimulus. It could be that the additional cortical activity generated by the occurrence of the lexical change was not strong enough to involuntarily engage attention, and thus did not distract from the primary task, or alternatively, that physical and lexical information are processed in parallel [23].

\section{Conclusion}

This study demonstrates that, similar to rare acoustic deviants, higher-order deviants such as lexical changes are detected automatically. Our data suggest that a common process is involved in the detection of lexical change, independent of the strength of the LTM network linked to the standard and deviant stimuli. An interpretation consistent with this general change detection mechanism is that, following the lexical analysis of the stimuli, a mental representation of the lexical regularity is formed, and used as a basis for the detection of lexical change.

\section{Acknowledgements}

Supported by a research fellowship from the Alexander von Humboldt foundation to A.M.G., and a grant from the Deutsche Forschungsgemeinschaft.

\section{References}

1. Näätänen R. The role of attention in auditory information processing as revealed by event-related potentials and other brain measures of cognitive function. Behav Brain Sci 1990; 13:201-288.

2. Schröger E, Wolff C. Behavioral and electrophysiological effects of taskirrelevant sound change: a new distraction paradigm. Cogn Brain Res 1998; 7:71-87.

3. Pulvermüller F, Kujala T, Shtyrov Y, Simola J, Tiitinen H, Alku P, et al. Memory traces for words as revealed by the mismatch negativity. NeuroImage 2001; 14:607-616.

4. Pulvermüller F, Shtyrov Y, Kujala T, Näätänen R. Word-specific cortical activity as revealed by the mismatch negativity. Psychophysiology 2004; 41:106-112.

5. Shtyrov Y, Pihko E, Pulvermüller F. Determinants of dominance: is language laterality explained by physical or linguistic features of speech? NeuroImage 2005; 27:37-47.

6. Shtyrov Y, Pulvermüller F. Neurophysiological evidence of memory traces for words in the human brain. NeuroReport 2002; 13:521-525.

7. Jacobsen T, Horvath J, Schröger E, Lattner S, Widmann A, Winkler I. Preattentive auditory processing of lexicality. Brain Lang 2004; 88:54-67.

8. Diesch E, Biermann S, Luce T. The magnetic mismatch field elicited by words and phonological non-words. NeuroReport 1998; 9:455-460.

9. Escera C, Yago E, Corral MJ, Corbera S, Nunez MI. Attention capture by auditory significant stimuli: semantic analysis follows attention switching. Eur J Neurosci 2003; 18:2408-2412. 
10. Jacobsen T, Schröger E, Winkler I, Horvath J. Familiarity affects the processing of task-irrelevant auditory deviance. J Cogn Neurosci 2005; 17:1704-1713.

11. Frangos J, Ritter W, Friedman D. Brain potentials to sexually suggestive whistles show meaning modulates the mismatch negativity. NeuroReport 2005; 16:1313-1317.

12. Shtyrov Y, Osswald K, Pulvermüller F. Memory Traces for spoken words in the brain as revealed by the hemodynamic correlate of the mismatch negativity. Cereb Cortex 2007; doi:10.1093/cercor/bhm028.

13. Luce PA, Pisoni DB. Recognizing spoken words: the neighborhood activation model. Ear Hear 1998; 19:1-36.

14. Taft M. Lexical access codes in visual and auditory word recognition. Lang Cogn Process 1986; 1:297-308.

15. Meftah M, Boudelaa S. How facilitatory can lexical information be during word recognition? Evidence from Moroccon Arabic. Proc ICSLP 1996; 1:74-77

16. Ganong WF. Phonetic categorization in auditory word perception. J Exp Psychol Hum Percept Perform 1980; 6:110-125.
17. Pitt MA. The locus of the lexical shift in phoneme identification. I Exp Psychol Learn Mem Cogn 1995; 21:1037-1052.

18. Vitevitch MS, Luce PA. When words compete: levels of processing in perception of spoken words. Psychol Sci 1998; 9:325-329.

19. Price CJ, Wise RJ, Frackowiak RS. Demonstrating the implicit processing of visually presented words and pseudowords. Cereb Cortex 1996; 6: 62-70.

20. Pulvermüller F, Mohr B, Lutzenberger W. Neurophysiological correlates of word and pseudo-word processing in well-recovered aphasics and patients with right-hemispheric stroke. Psychophysiology 2004; 41: 584-591.

21. Sereno SC, Rayner K, Posner MI. Establishing a time-line of word recognition: evidence from eye movements and event-related potentials. NeuroReport 1998; 9:2195-2200.

22. Attias J, Pratt H. Auditory event related potentials during lexical categorization in the oddball paradigm. Brain Lang 1992; 43:230-239.

23. Kotchoubey B, Lang S. Parallel processing of physical and lexical auditory information in humans. Neurosci Res 2003; 45:369-374. 